\title{
LOS PLECÓPTEROS (INSECTA, PLECOPTERA) DE LA CUENCA DEL RÍO SEGURA (S.E. DE ESPAÑA): 1. ESTUDIO FAUNÍSTICO
}

\author{
N. A. Ubero-Pascal (*), M. A. Puig (**) y A. G. Soler (*)
}

\begin{abstract}
RESUMEN
En este trabajo se presentan los primeros datos de los Plecópteros para el conjunto de la cuenca del río Segura. El trabajo se basa fundamentalmente en los datos obtenidos en muestreos llevados a cabo entre los años 1993 y 1994. Como complemento a éstos se han utilizado también los datos inéditos procedentes de la colección del Departamento de Biología Animal de la Universidad de Murcia y los obtenidos de la revisión bibliográfica.

Palabras clave: Plecoptera, Faunística, Distribución, Cuenca del río Segura, S.E. Ibérico.
\end{abstract}

\begin{abstract}
The stoneflies (Insecta, Plecoptera) from the Segura river basin: 1. Faunistic study

The fauna of Plecoptera in the Segura river basin is shortly known; only some references on this subject can be found in lymnological papers. Here we have performed the first check list of stoneflies of the Segura river basin. This papers is mainly based in samplings carried out between 1993 and 1994. Complementary we have used umpublished data from the stonefly collection of the Departamento de Biología Animal (Universidad de Murcia) and data from literature.
\end{abstract}

Keywords: Plecoptera, Faunistic, Distribution, Segura basin, South-western Spain.

\section{Introducción}

Los Plecópteros son uno de los principales grupos de macroinvertebrados de los ecosistemas lóticos dulceacuícolas (Salveit et al., 1987) y tienen una gran importancia en estudios de biología aplicada (Alba-Tercedor \& Sánchez-Ortega, 1988; Salveit et al., 1987), ya que se caracterizan por presentar unos requerimientos ambientales muy concretos (Hynes, 1976), siendo en la mayoría de los casos muy sensibles a cambios en su hábitat. Este hecho, junto a la multitud de agresiones que se están realizando sobre los sistemas fluviales ibéricos, puede provocar la desaparición de los mismos sin que lleguen a ser conocidos, por lo que se plantea la urgente necesidad de su estudio en aquellos sistemas fluviales donde aún no se sabe nada de ellos.

La cuenca del río Segura es una de las cuencas que más agresiones ha estado sufriendo en los últimos años, tanto de manera natural, por la sequía, como de una manera antrópica por la regulación de su cauce. El conocimiento de su fauna de Plecópteros es muy escaso, ya que tan sólo existen algunas citas en Aubert (1963a) y Vidal-Abarca et al. (1990), por lo que urgía un estudio detallado (Ubero-Pascal, 1996).

\footnotetext{
* Departamento de Biología Animal (Zoología). Universidad de Murcia. Campus de Espinardo. 30100. Murcia
}

** Centro de Estudios Avanzados de Blanes (C.S.I.C.). Camino de Santa Bárbara s/n. 17300 Blanes. Gerona. 




Fig. 1.- Localización geográfica del área de estudio, donde se encuentran señaladas las estaciones de muestreo del periodo 1993-94.

Fig. 1.- Geographic situation of study area, where have been placed the sampling stations of 1993-94 period.

En el presente estudio se ha intentado dar una primera visión de la riqueza faunística de Plecópteros que presenta la cuenca del río Segura, así como su distribución, de manera que contribuya a aumentar el conocimiento global de la fauna de macroinvertebrados de la misma y de la Península Ibérica.

\section{Material y Métodos}

La cuenca del río Segura se encuentra ubicada en el sudeste de la Península Ibérica (fig. 1), ocupando una área de $19.525 \mathrm{~km}^{2}$ que se extiende ampliamente por las provincias de Murcia y Albacete y, en menor medida, por las provincias de Alicante, Almería, Granada y Jaén.
Para llevar a cabo el estudio se han elegido 24 localidades de muestreo, de manera que quedaran representados adecuada y sintéticamente los distintos ambientes lóticos de la cuenca del río Segura (Tabla I). Para la elección de algunas de estas localidades de muestreo se utilizaron datos previos de capturas de Plecópteros.

El estudio se ha efectuado entre julio de 1993 y julio de 1994. Dicho estudio ha comprendido dos tipos de muestreo distintos: uno periódico, con una frecuencia trimestral y donde se capturaron tanto ninfas como imagos; y otro puntual, en dos ocasiones (otoño y primavera), donde sólo se capturaron imagos. Los datos de las muestras de invierno no se han tenido en cuenta ya que los especímenes encontrados eran demasiados pequeños, su deter- 
Tabla I. - Lista de localidades de muestreo (1993-94).

Table I._ List of sampling station (1993-94).

\begin{tabular}{|c|c|c|c|c|}
\hline Código & Localidades de Muestreo & Provincia & U.T.M. & Altituo \\
\hline L1 & Arroyo del Tús & $A B$ & $30 \mathrm{SWH} 507520$ & 1300 \\
\hline L2 & Río Zumeta en Santiago de la Espada & $J$ & $30 \mathrm{SWH} 388177$ & 1245 \\
\hline L3 & Río Taibilla en Nerpio & $A B$ & $30 S W H 633249$ & 1230 \\
\hline L4 & Río Madera cerca de Los Negros & $\mathrm{J}$ & 30 SWH335334 & 1180 \\
\hline L5 & Arroyo de La Celada & $A B$ & 30 SWH565587 & 1000 \\
\hline L6 & Río Mundo cerca de Los Chorros & $A B$ & $30 \mathrm{SWH} 498587$ & 985 \\
\hline L7 & Río Benamor en El Somogil & MU & $30 S W H 872299$ & 870 \\
\hline L8 & Río Mundo en Ayna & $A B$ & $30 \mathrm{SWH} 785675$ & 750 \\
\hline L9 & Río Mula en su Nacimiento & MU & $30 \mathrm{SXH148091}$ & 660 \\
\hline L10 & Río Mula en el Salto del Lucero & MU & $30 \mathrm{SXH} 165097$ & 615 \\
\hline L11 & Río Segura en La Graya & $A B$ & 30 SWH572405 & 615 \\
\hline L12 & Rambla de Fuente Álamo & MU & $30 S \times G 279546$ & 570 \\
\hline L13 & Río Corneros en La Fuensanta & MU & $30 S W G 961750$ & 550 \\
\hline L14 & Río Mundo antes del embalse del Talave & $A B$ & 30SWH939633 & 525 \\
\hline L15 & Rambla de Malvariche & MU & $30 S X G 969239$ & 515 \\
\hline L16 & Río Segura en Las Minas & $A B$ & 30SXH155422 & 310 \\
\hline L17 & Río Pliego cerca de Pliego & MU & $30 \mathrm{SXH} 318090$ & 300 \\
\hline L18 & Río Segura en Calasparra & MU & $30 \mathrm{SXH} 147340$ & 260 \\
\hline L19 & Rambla del Puerto de La Cadena & MU & 30SXG624972 & 260 \\
\hline L20 & Rambla del Judío & MU & $30 \mathrm{SXH} 366402$ & 250 \\
\hline L21 & Rambla del Moro & MU & $30 \mathrm{SXH} 436336$ & 240 \\
\hline L22 & Río Segura antes de Cieza & MU & $30 \mathrm{SXH333337}$ & 190 \\
\hline L23 & Rambla salada de Fortuna & MU & $30 \mathrm{SXH635222}$ & 140 \\
\hline L24 & Río Segura antes de Guardamar & A & 30SXH021192 & 6 \\
\hline
\end{tabular}

minación podría inducir a error, y las especies determinadas estaban ampliamente representadas en las tres muestras restantes.

Para la toma de muestras, tanto del bentos como del medio aéreo, se siguió un método semicuantitativo por unidad de tiempo (10 minutos), utilizándose una manga triangular para capturar el bentos y una manga circular para el medio aéreo. La técnica de muestreo seguida para la captura del bentos es la descrita por Armitage (1984). Las muestras de bentos se conservaron en formaldehído al 4\% y las aéreas en etanol al $70 \%$.

Se han utilizado también los datos extraídos de la colección del Departamento de Biología Animal de la Universidad de Murcia. Dicho material se encuentra actualmente depositado en las colecciones del Departamento de Ecología Fluvial del Centro de Estudios Avanzados de Blanes. En las etiquetas de localización las localidades de muestreo venían referidas mediante códigos de campo, por lo tanto ha sido necesario su descodificación. La caracterización de estas localidades se ha podido realizar usando las claves de códigos que exis- ten y mediante la consulta de los listados de localidades de muestreo que aparecen en distintos trabajos (Gómez, 1988; Vidal-Abarca, 1985). La relación de estas localidades puede consultarse en la Tabla II. Todas las citas referentes a esta colección se han señalado con un asterisco.

Complementando a estos datos se ha realizado una revisión bibliográfica incorporando en cada taxón la relación de citas para la cuenca del río Segura.

\section{Resultados}

En la ordenación de los resultados se ha seguido la que aparece en Zwick (1973) y Sánchez-Ortega \& Alba-Tercedor (1987).

Familia Perlodidae Klapalek, 1912 Guadalgenus franzii (Aubert, 1963)

Material estudiado: 30SWH939633; 20/III/94; Río Segura en La Graya, Yeste, Albacete; 615 m.; 9 ninfas. 
Tabla II. - Lista de las localidades del material procedente de las colecciones del Departamento de Biología Animal.

Table II. - List of sampling station of specimens from Biología Animal Department collection.

\begin{tabular}{|c|c|c|c|c|}
\hline Código & Localidades de Muestreo & Provincia & U.T.M. & Altitud \\
\hline M-1 & Río Segura en su nacimiento & $J$ & $30 \mathrm{SWH} 2717$ & 1400 \\
\hline M-2 & Río Madera & $\mathrm{J}$ & $30 \mathrm{SWH} 3738$ & 1250 \\
\hline M-3 & Río Mundo, Arroyo de la Celada & $A B$ & 30SWH5658 & 900 \\
\hline M-4 & Río Taibilla en El Peñón & $A B$ & 30 SWH6424 & 950 \\
\hline M-5 & Río Mula, Rambla del Ceacejo. & MU & $30 \mathrm{SXH} 1309$ & 500 \\
\hline M-6 & Las Fuentes del río Mula & MU & $30 \mathrm{SXH} 1409$ & 490 \\
\hline$M-7$ & R. Mula a $200 \mathrm{~m}$ de las Fuentes del R. Mula & MU & $30 \mathrm{SXH} 1409$ & 450 \\
\hline M-8 & R. Mula frente a las Casas de Ucenda. & MU & $30 \mathrm{SXH} 1609$ & 400 \\
\hline M-9 & R. Mula en El Salto del Lucero & MU & $30 \mathrm{SXH} 1709$ & 400 \\
\hline$M-10$ & R. Mula en el Molino de Sebastián & MU & $30 \mathrm{SXH} 1810$ & 350 \\
\hline$M-11$ & R. Mula en el puente de los ocho ojos. & MU & $30 \mathrm{SXH} 2010$ & 300 \\
\hline$M-12$ & R. Mula en el Km. 32 de la carretera Mula-Bullas & MU & $30 \mathrm{SXH} 2812$ & 280 \\
\hline $\mathrm{M}-13$ & R. Mula, Fuente del Pilar en Campos del Río & MU & $30 \mathrm{SXH} 4511$ & 165 \\
\hline $\mathrm{M}-14$ & R. Mula en Campos del Río & MU & $30 \mathrm{SXH} 4511$ & 165 \\
\hline M-15 & R. Mula, Fuente Caputa & MU & $30 \mathrm{SXH} 3116$ & 460 \\
\hline M-16 & R. Mula, Rambla de Ucenda & MU & 30SXH1508 & 450 \\
\hline $\mathrm{M}-17$ & Canal que desde Ucenda va al R. Mula & MU & $30 \mathrm{SXH} 1404$ & 400 \\
\hline$M-18$ & R. Mula, Rambla de Perea & MU & $30 \mathrm{SXH} 3215$ & 410 \\
\hline M-19 & R. Pliego, Rambla de Zarzadilla en Casas Nuevas & S MU & $30 S X G 2498$ & 450 \\
\hline
\end{tabular}

Esta especie había sido citada anteriormente en la cuenca del río Segura como Isogenus franzii (Aubert, 1963a) por Vidal-Abarca et al. (1990). Su distribución se encuentra muy localizada en el tramo medio-alto de la cuenca.

\section{Isoperla bipartita Aubert, 1962}

De esta especie sólo se conoce para la cuenca del río Segura la cita de Vidal-Abarca et al. (1990).

\section{Isoperla grammatica (Poda 1761)}

Material estudiado: 30SWH335334; 27/XI/1993; Río Madera cerca de Los Negros, Orcera, Jaén; 1180 m.; 1 ninfa. 30SWH335334; 19/III/1994; Río Madera cerca de Los Negros, Orcera, Jaén; 1180 m.; 39 ninfas. 30SWH565587; 30/V/1994; Arroyo de la Celada, Riopar, Albacete; 1000 m.;1 imago o". 30SWH498587; 19/III/1994; Río Mundo cerca de Los Chorros, Riopar, Albacete; 985 m.; 1 ninfa.

De esta especie se conocen las citas para la cuenca de Aubert (1963) y de Vidal-Abarca et al. (1990). Se distribuye exclusivamente por el tramo alto de la cuenca.

\section{Isoperla pallida Aubert, 1963}

Material eStudiado: 30SWH388177; 20/III/1994; Río Zumeta en Santiago de la Espada, Santiago de la Espada, Jaén; 1245 m.; 12 ninfas. 30SWH565587; 19/III/1994; Arroyo de La Celada, Riopar, Albacete; 1000 m.; 34 ninfas. 30SWH498587; 19/III/1994; Río Mundo cerca de Los Chorros, Riopar, Albacete; 985 m.; 2 ninfas.

En la cuenca del río Segura se conoce la cita de Vidal-Abarca et al. (1990). Esta especie se distribuye exclusivamente por el tramo alto de la cuenca.

Familia Perlidae Latreille, 1802 Dinocras cephalotes (Curtis, 1827)

Material estudiado: 30SWH388177; 28/XI/1993; Río Zumeta en Santiago de la Espada, Santiago de la Espada, Jaén; 1245 m.; 17 ninfas. 30SWH388177; 20/III/1994; Río Zumeta en Santiago de la Espada, Santiago de la Espada, Jaén; 1245 m.; 37 ninfas. 30SWH498587; 10/VII/1993; Río Mundo cerca de Los Chorros, Riopar, Albacete; 985 m.; 2 ninfas. *30SXH1309; 20/VIII/1982; Rambla del Ceacejo, Bullas, Murcia; 500 m.; 2 ninfas.

De esta especie se conoce la cita para la cuenca de Vidal-Abarca et al. (1990). Se distribuye exclusivamente por el tramo alto de la cuenca. 
Eoperla ochracea (Kolbe, 1885)

Material estudiado: 30SWH872299; 27/XI/1993; Río Benamor en El Somogil, Moratalla, Murcia; 870 m.; 1 ninfa. 30SWH872299; 25/III/1994; Río Benamor en El Somogil, Moratalla, Murcia; 870 m.; 1 ninfa. 30SWH872299; 24/V/1994; Río Benamor en El Somogil, Moratalla, Murcia; 870 m.; 5 imagos $0^{x} 0^{x}$ y 2 imagos 우 ㅇ․ 30SXH148091; 24/XI/1993; Río Mula en su nacimiento, Bullas, Murcia; 660 m.; 9 ninfas. 30SXH165097; 24/III/1994; Río Mula en el Salto del Lucero, Bullas, Murcia; 615 m.; 3 ninfas. *30SWH5658; 28/VIII/1982; Arroyo de la Celada, Riopar, Albacete; 900 m.; 1 ninfa. *30SXH1409; 18/II/1981; Las Fuentes del Río Mula, Bullas, Murcia; 490 m.; 2 ninfas. *30SXH1409; 18/III/1981; Río Mula a $200 \mathrm{~m}$. de las Fuentes del Río Mula, Bullas, Murcia; 450 m.; 1 ninfa. *30SXH1409; 18/IV/1981; Río Mula a $200 \mathrm{~m}$. de las Fuentes del Río Mula, Bullas, Murcia; 450 m.; 1 ninfa. *30SXH1609; 28/I/1980; Río Mula frente a las Casas de Ucenda, Bullas, Murcia; 400 m.; 1 ninfa. *30SXH1609; 1/XI/1981; Río Mula frente a las Casas de Ucenda, Bullas, Murcia; 400 m.; 11 ninfas. *30SXH1609; 19/XII/1981; Río Mula frente a las Casas de Ucenda, Bullas, Murcia; 400 m.; 15 ninfas. *30SXH1609; 31/I/1982; Río Mula frente a las Casas de Ucenda, Bullas, Murcia; 400 m.; 5 ninfas. *30SXH1609; 6/IV/1982; Río Mula frente a las Casas de Ucenda, Bullas, Murcia; 400 m.; 17 ninfas. *30SXH1609; 20/V/1982; Río Mula frente a las Casas de Ucenda, Bullas, Murcia; 400 m.; 9 ninfas. *30SXH1609; 20/VI/1982; Río Mula frente a las Casas de Ucenda, Bullas, Murcia; 400 m.; 2 ninfas. *30SXH1709; 11/III/1981; Río Mula en el Salto del Lucero, Bullas, Murcia; 400 m.; 1 ninfa. *30SXH1810; 11/III/1981; Río Mula en el Molino de Sebastián, Bullas, Murcia; 350 m.; 8 ninfas. *30SXH4511; 18/II/1981; R. Mula, Fuente del Pilar en Campos del Río, Campos del Río, Murcia; 165 m.; 1 ninfa. *30SXH4511; 22/XI/1980; R. Mula en Campos del Río, Campos del Río, Murcia; 165 m.; 109 ninfas. *30SXH3116; 18/IX/1980; Fuente Caputa, Mula, Murcia; 460m.; 2 ninfas. *30SXH1404; 27/V/1981; Canal que desde Ucenda va al R. Mula, Bullas, Murcia; 400 m.; 1 ninfa. *30SXH3215; 9/X/1980; Rambla de Perea, Mula, Murcia; 410 m.; 28 ninfas. *30SXG2498; 11/III/1981; Rambla de Zarzadilla en Casas Nuevas, Mula, Murcia; 450 m.; 3 ninfas.

La presencia de esta especie en la cuenca del río Segura constituye una nueva cita para la misma, distribuyéndose ampliamente por su tramo medio.

\section{Perla grandis Rambur, 1842}

Material estudiado: *30SWH2717; 21/VIII/1982; Río Segura en su nacimiento, Pontones, Jaén; 1400 m.; 1 imago 우 .

Esta especie también constituye una nueva cita para la cuenca del río Segura, presentado una distribución escasa y muy localizada en el tramo alto de la misma.
Perla marginata (Panzer, 1799)

MATERIAL ESTUDIADO: 30SWH507520; 28/XI/1993; Arroyo del Tus, Yeste, Albacete; 1300 m.; 137 ninfas. 30SWH507520; 20/III/1994; Arroyo del Tus, Yeste, Albacete; 1300 m.; 97 ninfas. 30SWH507520; 10/VII/1993; Arroyo del Tus, Yeste, Albacete; 1300 m.; 47 ninfas y 1 imago ơ. 30SWH335334; 27/XI/1993; Río Madera cerca de Los Negros, Orcera, Jaén; 1180 m.; 56 ninfas. 30SWH335334; 19/III/1994; Río Madera cerca de Los Negros, Orcera, Jaén; 1180 m.; 6 ninfas. 30SWH335334; 10/VII/1993; Río Madera cerca de Los Negros, Orcera, Jaén; 1180 m.; 14 ninfas. 30SWH565587; 27/XI/1993; Arroyo de La Celada, Riopar, Albacete; 1000 m.; 5 ninfas. 30SWH565587; 19/III/1994; Arroyo de La Celada, Riopar, Albacete; 1000 m.; 11 ninfas. 30SWH565587; 10/VII/1993; Arroyo de La Celada, Riopar, Albacete; 1000 m.; 14 ninfas. 30SWH498587; 27/XI/1993; Río Mundo cerca de Los Chorros, Riopar, Albacete; 985 m.; 10 ninfas. 30SWH498587; 19/III/1994; Río Mundo cerca de Los Chorros, Riopar, Albacete; 985 m.; 15 ninfas. 30SWH498587; 10/VII/1993; Río Mundo cerca de Los Chorros, Riopar, Albacete; 985 m.; 2 ninfas. *30SWH3738; 22/VIII/1982; Río Madera, Orcera, Jaén; 1250 m.; 2 ninfas. *30SWH6424; 20/VIII/1982; Río Taibilla en El Peñón, Nerpio, Albacete; 950 m.;1 ninfa.

Para la cuenca del río Segura se conocen la citas de Aubert (1963a) y de Vidal-Abarca et al. (1990). Esta especie se distribuye ampliamente y casi con exclusividad por el tramo alto de la cuenca, aunque también puede alcanzar el tramo medio.

Familia Nemouridae Klapalek, 1905 Protonemura beatensis (Despax, 1929)

De esta especie sólo se conoce la cita para la cuenca del río Segura de Vidal-Abarca et al. (1990).

\section{Protonemura meyeri (Pictet, 1841)}

Material eStudiado: 30SWH507520; 28/XI/1993; Arroyo del Tus, Yeste, Albacete; 1300 m.; 57 ninfas. 30SWH507520; 20/III/1994; Arroyo del Tus, Yeste, Albacete; 1300 m.; 6 ninfas. 30SWH388177; 28/XI/1993; Río Zumeta en Santiago de la Espada, Santiago de la Espada, Jaén; 1245 m.; 3 ninfas. 30SWH388177; 20/III/1994; Río Zumeta en Santiago de la Espada, Santiago de la Espada, Jaén; 1245 m.; 1 ninfa. 30SWH335334; 27/XI/1993; Río Madera cerca de Los Negros, Orcera, Jaén; 1180 m.; 45 ninfas. 30SWH335334; 19/III/1994; Río Madera cerca de Los Negros, Orcera, Jaén; 1180 m.; 5 ninfas. 30SWH565587; 27/XI/1993; Arroyo de La Celada, Riopar, Albacete; 1000 m.; 51 ninfas. 30SWH565587; 19/III/1994; Arroyo de La Celada, Riopar, Albacete; 1000 m.; 2 ninfas. 30SWH565587; 10/VII/1993; Arroyo de La Celada, Riopar, Albacete; 1000 m.; 6 ninfas. 30SWH498587; 27/XI/1993; Río Mundo cerca de Los Chorros, Riopar, Albacete; 985 m.; 1 ninfa. 
Esta especie había sido citada anteriormente en la cuenca por Vidal-Abarca et al. (1990). Se encuentra distribuida amplia y exclusivamente por el tramo alto de la cuenca.

\section{Protonemura cf. pyrenaica Aubert, 1954}

Material estudiado: 30SWH565587; 27/XI/1993; Arroyo de La Celada, Riopar, Albacete; 1000 m.; 2 ninfas.

En la cuenca del río Segura se conoce la cita de esta especie de Vidal-Abarca et al. (1990). Se encuentra exclusivamente en el tramo alto de la cuenca.

\section{Nemoura fulviceps Klapalek, 1902}

MATERIAl ESTUDIADO: 30SWH507520; 28/XI/1993; Arroyo del Tus, Yeste, Albacete; 1300 m.; 1 imago ㅇ․ 30SWH507520; 13/XI/1994; Arroyo del Tus, Yeste, Albacete; 1300 m.; 1 imago ㅇ . 30SWH388177; 20/III/1994; Río Zumeta en Santiago de la Espada, Santiago de la Espada, Jaén; 1245 m.; 6 ninfas. 30SWH388177; 10/VII/1993; Río Zumeta en Santiago de la Espada, Santiago de la Espada, Jaén; 1245 m.; 11 ninfas. 30SWH633249; 19/VII/1994; Río Taibilla en Nerpio, Nerpio, Albacete; 1230 m.; 4 ninfas. *30SXH1609; 31/VIII/1981; R Mula frente a las casas de Ucenda, Bullas, Murcia; 400 m.; 1 ninfa. *30SXH1810; 11/III/1981; Río Mula en el Molino de Sebastián, Bullas, Murcia; 350 m.; 2 ninfas. *30SXH2010; 11/III/1981; Río Mula en el puente de los ocho ojos, Bullas, Murcia; 300 m.; 18 ninfas. *30SXH2812; 16/III/1981; Río Mula en el Km. 32 de la carretera Mula-Bullas, Mula, Murcia; 280 m.; 2 ninfas y 1 imago 우.

Esta especie había sido citada para la cuenca del río Segura como Nemoura navasi Aubert, 1953 (Aubert, 1963a). Se distribuye tanto por el tramo alto como por el tramo medio de la cuenca.

\section{Nemoura lacustris Pictet, 1865}

Material ESTUdiado: 30SWH388117; 20/III/1994; Río Zumeta en Santiago de la Espada, Santiago de la Espada, Jaén; 1245 m.; 4 ninfas. 30SWH633249; 19/VII/1994; Río Taibilla en Nerpio, Nerpio, Albacete; 1230 m.; 1 ninfa. *30SXH2812; 26/III/1981; Río Mula en el Km. 32 de la carretera MulaBullas, Mula, Murcia; 280 m.; 3 ninfas.

Esta especie había sido citada anteriormente en la cuenca del río Segura por Aubert (1963a) y por Vidal-Abarca et al. (1990). Presenta una distribución exclusiva por las localidades de muestreo de mayor altitud del tramo alto de la cuenca, aunque también se tienen datos de su presencia en el tramo medio.

\section{Nemoura ef. mortoni Ris, 1902}

MATERIAL ESTUDIADO: 30SWH388177; 28/XI/1993; Río Zumeta en Santiago de la Espada, Santiago de la Espada, Jaén; 1245 m.; 1 ninfa. *30SXH1609; 1/VII/1981; Río Mula frente a las casas de Ucenda, Bullas, Murcia; 400 m.; 1 ninfa. *30SXH1508; 27/V/1981; Rambla de Ucenda, Bullas, Murcia; 280 m.; 1 ninfa.

La captura de esta especie constituye una nueva cita para la cuenca del río Segura y se encuentra distribuida tanto por su tramo alto como por su tramo medio.

\section{Familia Capniidae Klapalek, 1905 Capnioneura mitis Despax, 1932}

Material estudiado: 30SWH388177; 28/XI/1993; Río Zumeta en Santiago de la Espada, Santiago de la Espada, Jaén; 1245 m.; 8 ninfas. 30SWH388177; 20/III/1994; Río Zumeta en Santiago de la Espada, Santiago de la Espada, Jaén; 1245 m.; 1 imago O". 30SWH335334; 27/XI/1993; Río Madera cerca de Los Negros, Orcera, Jaén; 1180 m.; 182 ninfas. 30SWH335334; 19/III/1994; Río Madera cerca de Los Negros, Orcera, Jaén; 1180 m.; 2 imagos 우 ㅇ․ 30SWH565587; 27/XI/1993, Arroyo de La Celada, Riopar, Albacete; 1000 m.; 39 ninfas. 30SWH498587; 27/XI/1993; Río Mundo cerca de Los Chorros, Riopar, Albacete; 985 m.; 10 ninfas y 1 imago o". 30SWH939633; 27/XI/1993; Río Mundo antes del Embalse del Talave, Lietor, Albacete; 525 m.; 2 imagos $0^{7} \sigma^{7}$.

En la cuenca del río Segura se conoce la cita para esta especie de Vidal-Abarca et al. (1990). Presenta una amplia distribución por el tramo alto de la cuenca, aunque también se ha capturado en una localidad de muestreo del tramo medio.

Familia Leuctridae Klapalek, 1905 Leuctra andalusiaca Aubert, 1962

MATERIAL ESTUDIADO: 30SWH507520; 20/III/1994; Arroyo de Tus, Yeste, Albacete; 1300 m.; 1 ninfas. 30SWH388177; 20/III/1994; Río Zumeta en Santiago de la Espada, Santiago de la Espada, Jaén; 1245 m.; 1 imago $\sigma^{\pi}$ y 1 imago $\sigma^{\pi}$. 30SWH335334; 19/III/1994; Río Madera cerca de Los Negros, Orcera, Jaén; 1180 m.; 2 ninfas. 30SWH498587; 19/III/1994; Río Mundo cerca de Los Chorros, Riopar, Albacete; 985 m.; 9 ninfas.

La captura de esta especie constituye una nueva cita para la cuenca del río Segura y se distribuye exclusivamente por el tramo alto de la cuenca. 


\section{Leuctra aurita Navás, 1919}

MATERIAL ESTUDIADO: 30SWH507520; 28/XI/1993; Arroyo de Tus, Yeste, Albacete; 1300 m.; 258 ninfas. 30SWH507520; 10/VII/1993; Arroyo de Tus, Yeste, Albacete; 1300 m.; 272 ninfas. 30SWH388177; 28/XI/1993; Río Zumeta en Santiago de La Espada, Santiago de la Espada, Jaén; 1245 m.; 6 ninfas. 30SWH335334; 27/XI/1993; Río Madera cerca de Los Negros, Orcera, Jaén; 1180 m.; 2 ninfas. 30SWH565587; 10/VII/1993; Arroyo de La Celada, Riopar, Albacete; 1000 m.; 84 ninfas. 30SWH498587; 27/XI/1993; Río Mundo cerca de Los Chorros, Riopar, Albacete; 985 m.; 2 imagos 우 오. 30SWH872299; 22/XI/1993; Río Benamor en El Somogil, Moratalla, Murcia; 870 m.; 4 ninfas. 30SWH872299; 25/III/1994; Río Benamor en El Somogil, Moratalla, Murcia; 870 m.; 1 ninfas. 30SWH785675; 11/VII/1993; Río Mundo en Ayna, Ayna, Albacete; 750 m.; 4 ninfas.

En la cuenca del río Segura se conoce la cita para esta especie de Vidal-Abarca et al. (1990). Esta especie se encuentra ampliamente distribuida por todo el tramo alto de la cuenca, aunque también se ha encontrado en una de las localidades de muestreo de mayor altitud del tramo medio.

\section{Leuctra cazorlana Aubert, 1962}

Material estudiado: 30SWH335334; 16/XI/1993; Río Madera cerca de Los Negros, Orcera, Jaén; 1180 m.; 6 imagos $0^{7} \sigma^{7}$ y 4 imagos 우 ㅇ․ 30SWH335334; 12/XI/1994; Río Madera cerca de Los Negros, Orcera, Jaén; 1180 m.; 1 imago ㅇ. 30SWH498587; 27/XI/1993; Río Mundo cerca de Los Chorros, Riopar, Albacete; 985 m.; 1 imago $\sigma^{7}$ y 2 imagos 우 ㅇ․ . 30SWH498587; 17/XI/1994; Río Mundo cerca de Los Chorros, Riopar, Albacete; 985 m.; 1 imago $\sigma^{7}$. 30SWH939633; 17/XI/1993; Río Mundo antes del Embalse de El Talave, Lietor, Albacete; 525 m.; 1 imago $\sigma^{x}$.

Esta especie se encuentra distribuida por el tramo alto y medio de la cuenca del río Segura y esta cita es la primera para dicha cuenca.

\section{Leuctra espanoli Aubert, 1956}

De esta especie tan solo se conoce para la cuenca del río Segura la cita de Vidal-Abarca et al. (1990).

\section{Leuctra fusca (Linneo, 1758)}

Material estudiado: 30SWH335334; 13/XI/1994; Río Madera cerca de Los Negros, Orcera, Jaén; 1180 m.; 1 imago ơ. 30SWH335334; 10/VII/1993; Río Madera cerca de Los Negros, Orcera, Jaén; 1180 m.; 281 ninfas. 30SWH565587;
27/XI/1993; Arroyo de La Celada, Riopar, Albacete; 1000 m.; 1 imago o y 4 imagos 우 ㅇ․ 30SWH565587; 12/XI/1994; Arroyo de La Celada, Riopar, Albacete; 1000 m.; 5 imagos $0^{7} \sigma^{7}$ y 1 imago 우. 30SWH498587; 27/XI/1993; Río Mundo cerca de Los Chorros, Riopar, Albacete; 985 m.; 4 ninfas, 2 imagos $\sigma^{x} \sigma^{x}$ y 1 imago $ᄋ$. 30SWH498587; 10/VII/1993; Río Mundo cerca de Los Chorros, Riopar, Albacete; 985 m.; 238 ninfas. 30SWH872299; 22/XI/1993; Río Benamor en El Somogil, Moratalla, Murcia; $870 \mathrm{~m} . ; 1$ imago $\sigma^{7}$. 30SWH785675; 11/VII/1993; Río Mundo en Ayna, Ayna, Albacete; 750 m.; 1 ninfa. 30SWH572405; 20/VII/1994; Río Segura en La Graya, Yeste, Albacete; 615 m.; 7 ninfas.

De esta especie se conoce la cita de VidalAbarca et al. (1990). La distribución de esta especie se extiende desde el tramo alto hasta las localidades de mayor altitud del tramo medio de la cuenca.

\section{Leuctra geniculata Stephens, 1835}

Material estudiado: 30SWH335334; 10/VII/1993; Río Madera cerca de Los Negros, Orcera, Jaén; 1180 m.; 54 ninfas. 30SWH565587; 10/VII/1993; Arroyo de La Celada, Riopar, Albacete; 1000 m.; 30 ninfas. 30SWH565587; 27/XI/1993; Arroyo de La Celada, Riopar, Albacete; 1000 m.; 1 imago 우. 30SWH565587; 12/XI/1994; Arroyo de La Celada, Riopar, Albacete; 1000 m.; 1 imago O'. 30SWH498587; 10/VII/1993; Río Mundo cerca de Los Chorros, Riopar, Albacete; 985 m.; 28 ninfas. 30SWH872299; 8/VII/1994; Río Benamor en El Somogil, Moratalla, Murcia; 870 m.; 3 ninfas. 30SWH785675; 11/VII/1993; Río Mundo en Ayna, Ayna, Albacete; 750 m.; 2 ninfas. 30SWH572405; 20/VII/1994; Río Segura en La Graya, Yeste, Albacete; 615 m.; 59 ninfas.

Vidal-Abarca et al. (1990) cita esta especie en la cuenca del río Segura. La distribución de esta especie se extiende desde el tramo alto hasta las localidades de mayor altitud del tramo medio de la cuenca.

\section{Leuctra iliberis (Sánchez-Ortega y Alba-Tercedor, 1988)}

MATERIAL ESTUDIADO: 30SWH507520; 20/III/1994; Arroyo de Tus, Yeste, Albacete; 1300 m.; 1 imago $0^{x}$ y 2 imagos 우 우. 30SWH565587; 19/III/1994; Arroyo de La Celada, Riopar, Albacete; 1000 m.; 1 imago ㅇ․ 30SWH872299; 22/XI/1993; Río Benamor en El Somogil, Moratalla, Murcia; 870 m.; 2 imagos $\sigma^{7} \sigma^{7}$ y 2 imagos 우. 30SWH785675; 27/XI/1993; Río Mundo en Ayna, Ayna, Albacete; 750 m.; 1 ninfa.

La captura de esta especie constituye la primera cita para la cuenca del río Segura y su distribución es tanto por el tramo alto como por las localidades de mayor altitud del tramo medio de dicha cuenca. 
Leuctra cf. illiesi Aubert, 1956

MATERIAL ESTUDIADO: 30SWH565587; 27/XI/1993; Arroyo de La Celada, Riopar, Albacete; 1000 m.; 3 ninfas. *30SWH5658; 28/VIII/1982; Arroyo de la Celada, Riopar, Albacete; 900 m.; 1 ninfa.

Esta captura constituyen la primera cita de esta especie para la cuenca del río Segura y su distribución es exclusiva del tramo alto de dicha cuenca, siendo ésta muy localizada.

\section{Leuctra inermis Kempny, 1899}

Material estudiado: 30SWH335334; 27/XI/1993; Río Madera cerca de Los Negros, Orcera, Jaén; 1180 m.; 9 ninfas. 30SWH565587; 27/XI/1993; Arroyo de La Celada, Riopar, Albacete; 1000 m.; 115 ninfas. 30SWH872299; 8/VII/1994; Río Benamor en El Somogil, Moratalla, Murcia; 870 m.; 1 ninfas. 30SWH572405; 20/III/1994; Río Segura en La Graya, Yeste, Albacete; 615 m.; 10 ninfas.

Esta especie había sido citada con anterioridad en la cuenca del río Segura por Vidal-Abarca et al. (1990). Se distribuye tanto por el tramo alto como por las localidades de muestreo de mayor altitud del tramo medio.

\section{Leuctra cf. leptogaster Aubert, 1949}

Material eSTUDiado: 30SWH388177; 28/XI/193; Río Zumeta en Santiago de la Espada, Santiago de la Espada, Jaén; 1245 m.; 1 ninfa. 30SWH335334; 27/XI/1993; Río Madera cerca de Los Negros, Orcera, Jaén; 1180 m.; 7 ninfas. 30SWH498587; 27/XI/1993; Río Mundo cerca de Los Chorros, Riopar, Albacete; 985 m.; 14 ninfas. *30SXH3215; 9/X/1980; Rambla de Perea, Mula, Murcia; 410 m.; 1 ninfa.

En la cuenca del río Segura se conoce la cita de esta especie en Vidal-Abarca et al. (1990). Esta especie se distribuye fundamentalmente por el tramo alto de la cuenca, aunque puede alcanzar también al tramo medio-bajo.

\section{Leuctra maroccana Aubert, 1956}

Material estudiado: 30SWH335334; 13/XI/1994; Río Madera cerca de Los Negros, Orcera, Jaén; 1180 m.; 1 imago $O^{7}$.

La captura de esta especie constituye la primera cita para la cuenca del río Segura y su distribución se encuentra muy localizada en el tramo alto de dicha cuenca.

\section{Leuctra cf. stupeningi Illies, 1954}

Material eStudiado: 30SWH388177; 28/XI/1993; Río Zumeta en Santiago de la Espada, Santiago de la Espada, Jaén; 1245 m.; 3 ninfas.

Esta especie ha sido capturada por primera vez en la cuenca del río Segura, por lo que es una nueva cita para la misma, siendo su distribución muy localizada en el tramo alto de dicha cuenca.

\section{Tyrrhenoleuctra minuta (Klapalek, 1903)}

Esta especie ha sido citada por Aubert (1963a) en Sierra Espuña (Murcia).

\section{Discusión}

La fauna de Plecópteros de la cuenca del río Segura comprende 28 especies, englobadas en 10 géneros y 5 familias. La familia Leuctridae y, en concreto, el género Leuctra es el que presenta mayor número de especies, hecho que se ha podido comprobar en otras zonas (Membiela, 1996; Puig, 1984; Sánchez-Ortega \& Alba-Tercedor, 1990). Este hecho se puede deber a que es uno de los géneros de Plecópteros que más especies presenta en Europa (Pardo \& Zwick, 1993).

En cuanto a $P$. beatensis y L. espanoli, debido a la distribución que presentan en la Península Ibérica dichas especies (Sánchez-Ortega \& AlbaTercedor, 1987), la imposibilidad de revisar el material de las mismas y la ausencia de capturas propias, creemos que su presencia en la cuenca del río Segura puede ser dudosa. Por otro lado I. bipartita, aunque ha sido citada en el sur peninsular por Puig \& Ferreras (1983) y Aubert (1963a y b), por las razones anteriormente expuestas, creemos necesario poner también en duda su presencia en la cuenca, hasta que ésta pueda ser confirmada.

De las 24 especies capturadas, 9 son nuevas citas para la cuenca del río Segura. Debido a la baja densidad de capturas y a la distribución en la Península Ibérica (Sánchez-Ortega \& Alba-Tercedor, 1987) de las especies $P$. cf. pyrenaica, $N$ cf. mortoni, $L$. cf. illiesi, L. cf. Leptogaster y L. cf. stupeningi hemos creído conveniente nombrarlas de esta manera hasta poseer material suficiente o adultos que reconfirmen estas capturas, si bien $L$. cf. illiesi ya había sido citada en el sur de la Península (Puig \& Ferreras, 1983).

La presencia en la cuenca de las especies I. grammatica, I. pallida, D. cephalotes, $P$. grandis, P. mar- 
ginata, P. meyeri, Nemoura fulviceps, $N$. lacustris, $C$. mitis, L. aurita, L. fusca, L. geniculata, L. inermis y L. maroccana, viene a confirmar la amplia distribución de éstas en la Península Ibérica. En cuanto a $E$. ochracea, su captura en la cuenca del río Segura, contribuye a reafirmar su distribución circunmediterránea (Sánchez-Ortega \& Alba-Tercedor, 1987).

Por otro lado, la captura de G. franzi, L. andalusiaca, L. cazorlana y L. iliberis, endemismos de la Península Ibérica (Sánchez-Ortega \& AlbaTercedor, 1987), aumenta el área de distribución de éstas en la Península. En el caso de G. franzi al sudeste peninsular y en el caso de $L$. andalusiaca, $L$. cazorlana y L. iliberis, citadas solamente en distintas provincias andaluzas (Sánchez-Ortega \& AlbaTercedor, 1987; Ropero \& Sánchez-Ortega, 1992; Ropero et al., 1995), a la provincia de Albacete.

Los Plecópteros dentro de la cuenca del río Segura se encuentran distribuidos fundamentalmente por su tramo alto, si bien, algunas especies como: C. mitis, $N$. cf. mortoni, y la mayoría del género Leuctra, pueden aparecer en las localidades de mayor altitud del tramo medio. En cambio, $G$. franzi y E. ochracea, se encuentran distribuidas exclusivamente en el tramo medio de la misma.

El hecho de que algunas especies, como D. cephalotes y N. fulviceps, se hayan encontrado hace diez años en el tramo medio y que en la actualidad ya no se encuentren da idea de el grado de degradación que esta sufriendo la cuenca del río Segura en su tramo medio.

\section{Referencias}

Alba-Tercedor, J. \& Sánchez-Ortega, A., 1988. Un método rápido y simple para evaluar la calidad biológica de las aguas corrientes basado en el de Hellawell (1978). Limnética, 4: 51-56.

Armitage, P., 1984. Environmental changes induced by stream regulation and their effects on lotic macroinvertebrates communities. In: A. Lillehammer \& S. J. Salveit (eds.). Regulated Rivers. Universitetsforlaget. Oslo: 139-185.

Aubert, J., 1963a. Les Plécoptères de la Péninsule Ibérique. Eos, 39: 23-107.

Aubert, J., 1963b. Les Plécoptères des cours d'eau temporaires de la Péninsule Ibérique. Mitt. Schweiz. Entomol. Ges., 35: 301-315.

GómEz, R.M., 1988. Los Moluscos (Gastropoda \& Bivalvia) de las aguas continentales de la cuenca del río Segura (S.E. de España). Tesina de licenciatura. Facultad de Biología. Universidad de Murcia. 223 pp.

Hynes, H.B.N., 1976. Biology of Plecoptera. Ann. Rev. Entomol., 21: 135-153.
Membiela, P., 1996. Inventario dos Plecópteros de Galicia (Insecta: Plecoptera). Cadernos da Area de Ciencias Biolóxicas (Inventario). Seminario de Estudos Galegos, Vol. XIII. Ed. do Castro. A Coruña. 33 pp.

PARdo, I. \& ZWICK, P., 1993. Contribution to the knowledge of Mediterranean Leuctra (Plecoptera: Leuctridae). Bull. Soc. Entomol. Suisse, 66: 417-434.

Puig, M.A., 1984. Efemerópteros y Plecópteros de los Ríos Catalanes. Tesis Doctoral. Universidad de Barcelona. 582 pp.

Puig, M.A. \& Ferreras, M., 1983. Plecópteros de Sierra Morena central (Córdoba): datos preliminares. Actas I Congr. Ibér. Entomol., 2: 609-612.

Ropero, J.M. \& SÁnchez-Ortega, A., 1992. Descripción de la ninfa madura de Leuctra cazorlana Aubert, 1962 (Plecoptera, Leuctridae). Bol. Asoc. Esp. Entomol., 16: 43-49.

Ropero, J.M.; Peña, M.P. \& SÁnchez-Ortega, A., 1995. Composición y fenología de la fauna de Plecópteros (Insecta, Plecoptera) en el sur de España (Provincia de Cádiz). Bol. Asoc. Esp. Entomol., 19(3-4): 149-162.

Salveit, S.J.; Brittain, J.E. \& Lillehammer, A., 1987. Stoneflies and river regulation - A review. In: J. F. Craig \& J.B. Kemper (eds.). Regulated Streams. Plenum Publishing Corporation. New York: 117-129.

SÁncheZ-OrTEgA, A. \& AlbA-TERCEdor, J., 1987. Lista faunística y bibliográfica de los Plecópteros (Plecoptera) de la Península Ibérica. Asociación Española de Limnología. Madrid. 133 pp.

SÁncheZ-Ortega, A. \& AlbA-Tercedor, J., 1990. Los Plecópteros de Sierra Nevada (Granada, España). Zool. Baetica, 1: 77-146.

Ubero-Pascal, N.A. 1996. Estudio faunístico de los Órdenes Ephemeroptera y Plecoptera (Insecta) de la cuenca del río Segura (S.E. de la Península Ibérica). Tesina de Licenciatura. Facultad de Biología. Universidad de Murcia. 356 pp.

VIDAL-ABARCA, M.R. 1985. Las aguas superficiales de la cuenca del río Segura (S.E. de España). Caracterización físico-química en relación al medio físico y humano. Tesis Doctoral. Universidad de Murcia. 789 pp.

Vidal-Abarca, M.R.; SuÁrez, M. L.; Millán, A.; Gómez, R.; Ortega, M.; Velasco, J.; RamírezDíAZ, L. \& Montes, C., 1990. Estudio limnológico de la cuenca del río Mundo (Río Segura). Jornadas sobre el Medio Natural Albacetense: 339-357.

Zwick, P. 1973. Insecta: Plecoptera. Phylogenetische system und Katalog. Das Tierreich, 94: XXXII-645 pp.

Recibido, el 21-VII-1997

Aceptado, el 3-VII-1998

Publicado, el 31-XII-1998 